\title{
Diskussion ethischer Aspekte zahnärztlicher Altersschätzung bei jungen Flüchtlingen durch Röntgendiagnostik
}

\author{
Mathias Wirth • Christoph L. Menzel • Daniela C. M. Lee • \\ Heinz-Peter Schmiedebach
}

Eingegangen: 18. April 2016 / Angenommen: 7. November 2016 / Online publiziert: 23. Dezember 2016 (C) Springer-Verlag Berlin Heidelberg 2016

Zusammenfassung Eine zunehmende Zahl junger Flüchtlinge mit unklaren Altersangaben rückt das Problem der ärztlichen und zahnärztlichen Altersschätzungen durch Röntgenaufnahmen in den Fokus der öffentlichen Diskussion. Trotz anhaltender Kritik der organisierten deutschen Ärzteschaft an dieser Verfahrensweise und der hiermit verbundenen, nicht-medizinisch indizierten Strahlenexposition, ist es weiterhin gängige Praxis, zur Altersbestimmung Röntgenaufnahmen der Handwurzelknochen, des Schlüsselbeins oder der Zähne vorzunehmen. Dabei hat die amtliche Feststellung des Alters für die Asylsuchenden insofern erhebliche Auswirkungen, als minderjährigen Flüchtlingen besondere Rechte zukommen. Im bisherigen Diskurs über die Verhältnismäßigkeit der radiologischen Altersschätzung junger Asylsuchender wird zwar stets auf die Relevanz der Röntgenanalyse der dritten Molaren hingewiesen, die spezifische Rolle der Zahnärztinnen und Zahnärzte wird medizinethisch jedoch vergleichsweise wenig problematisiert. Tatsächlich hat die „Deutsche Gesellschaft für Zahn-, Mund- und Kieferheilkunde“ (DGZMK) in den Jahren 2001 und 2008 , wissenschaftliche Stellungnahmen“ publiziert, in denen eine vollumfängliche, radiologische Aufklärung des Alters von jungen Flüchtlingen empfohlen wird

Dr. phil. M. Wirth, Dipl. Theol. $(\bowtie)$

Institut für Geschichte und Ethik der Medizin, Zentrum für Psychosoziale Medizin, Universitätsklinikum Hamburg-Eppendorf, Martinistr. 52, 20246 Hamburg, Deutschland

E-Mail: m.wirth@uke.de

Dr. med. Dr. med. dent. C. L. Menzel, B.Sc.

Klinik für Mund-, Kiefer- und Gesichtschirurgie, Krankenhaus St. Johannes-Stift, Malteser Kliniken Rhein Ruhr, Duisburg, Deutschland

ZÄ D. C. M. Lee

Zahnarztpraxis Amler \& Mönch \& Knebel, Berlin, Deutschland

Prof. Dr. med. H.-P. Schmiedebach

Institut für Geschichte der Medizin und Ethik in der Medizin, (Professur für Medical Humanities),

Charité - Universitätsmedizin Berlin, Berlin, Deutschland 
- ohne dass eine ethische Problematisierung erfolgte. Vor dem Hintergrund dieser Befunde soll im Rahmen des vorliegenden Beitrags der Frage nachgegangen werden, inwieweit die in Deutschland etablierte zahnärztliche Praxis der Altersschätzung geeignet, erforderlich und ethisch angemessen ist. Methodische Grundlagen der Arbeit sind hierbei (1) eine umfassende Auswertung der verfügbaren Fachliteratur sowie (2) eine kritisch normative Analyse der maßgeblichen Argumentationslinien, wie sie insbesondere von der genannten Fachgesellschaft vertreten wird. Besonders in der ethischen Diskussion dieser Positionspapiere liegt ein Desiderat einer Ethik der Zahnmedizin und bietet Anlass, eine international geführte Debatte zur Altersbestimmung auf den konkreten Rahmen in Deutschland zu applizieren.

Schlüsselwörter Altersbestimmung bei minderjährigen Flüchtlingen · Röntgen in der Zahnmedizin · Güterabwägung · Berufsethos · Patientenwohl

\title{
Ethical discussion of dental age estimation in young refugees via $x$-ray diagnostics
}

\begin{abstract}
Definition of the problem An increasing number of young refugees whose ages are not reliably known is thrusting the problem of medical and dental age estimation via $\mathrm{x}$-ray examinations to the forefront of public discussion. Despite continual criticism of the organised German medical profession for this procedure and its associated radiation exposure for nonmedical purposes, $x$-raying the carpal bone, the clavicle or the teeth to determine age remains common practice. Consequently, the official verification of asylum seekers' ages has considerable ramifications since refugee minors have particular rights.

Arguments In previous discourse(s) about the proportionality of approximating the age of young asylum seekers via $\mathrm{x}$-rays, the relevance of the radiological examination of the wisdom teeth is indeed always referred to; however, the specific role of dentists is by comparison rarely discussed from a medical-ethical perspective. As a matter of fact, in 2001 and 2008 the Deutsche Gesellschaft für Zahn-, Mund- und Kieferheilkunde (DGZMK; English: German Association for Dentistry, Stomatology and Orthodontics) published scientific position papers in which the comprehensive radiological verification of the age of young refugees is endorsed - with the distinct omission of an ethical discussion.

Conclusion In light of these disparate findings, it should be investigated just how suitable, necessary and ethically appropriate dental procedures for the purposes of age determination are. The methodical foundations of this paper are (1) a comprehensive evaluation of available specialist literature, in addition to (2) a critical normative analysis of the main arguments presented by the named specialist society. Especially ethically discussing these position papers is a desideratum for ethics in dentistry. This discussion also serves as an opportunity to apply the international debate on age determination to Germany's framework.
\end{abstract}

Keywords Age determination in refugee minors $\cdot$ X-rays in dentistry $\cdot$ Weighing up choices $\cdot$ Professional ethos $\cdot$ Beneficence of patients 


\section{Einleitung}

Die Frage nach dem Umgang mit jungen, unbegleiteten Flüchtlingen stellen, heißt nicht, eine Diskussion über Marginalien zu führen (Bundesärztekammer 2016). Allein in Hamburg haben sich im Zeitraum von 2012 bis Mitte 2015 insgesamt 5415 unbegleitete, minderjährige Flüchtlinge bei den Behörden gemeldet. 1644 Personen sind hierbei zum Zweck der Altersbestimmung ärztlich und zahnärztlich untersucht worden (Focardi et al. 2014; Hillienhof 2015). Im gesamten europäischen Raum ist eine Zunahme der Altersschätzungen zu beobachten (Crawley 2007). Nicht allein aufgrund der großen Anzahl, sondern auch wegen der besonderen Hilfs- und Schutzbedürftigkeit junger Flüchtlinge sind die ethischen Implikationen derartiger Maßnahmen zu diskutieren. Die Altersschätzung ist insofern relevant, als minderjährigen Flüchtlingen ein besonderer Schutz zusteht, den die Jugendämter durch Inobhutnahme und Clearingverfahren garantieren sollen. Dieser besondere Schutz, verbunden mit besonderen Partizipationsrechten, wird volljährigen Flüchtlingen ab 18 Jahren nicht mehr gewährt (Riedelsheimer und Wiesinger 2004).

Außerdem ist zu erörtern, ob die kategorische Festlegung auf das 18. Lebensjahr als „Cut-off Punkt“ (Crawley 2007) einerseits und die Erhebung von Röntgenbefunden zur dentalen Altersbestimmung andererseits geeignet, erforderlich und ethisch angemessen sind. Diese beiden Fragen erhalten zusätzliche Brisanz, als es sich bei unbegleiteten, jungen Flüchtlingen um eine vulnerable Personengruppe handelt (Jensen et al. 2014; Keygnaert et al. 2012; Thevissen et al. 2012), die leicht Opfer von Ausbeutung, Gewalt und Unterdrückung werden kann. Dies gilt allerdings mutatis mutandis auch für bestimmte Gruppen erwachsener Asylsuchender, etwa für ältere Menschen (Strong et al. 2015).

Klärungsbedürftig ist überdies, ob die besagte diagnostische Maßnahme der Zahnärztin oder des Zahnarztes aus Sicht des betroffenen Flüchtlings nicht zugleich ein „Ich glaube Dir nicht“, „Ich helfe Dir nicht“ bzw. ein „Ich füge Dir Schaden zu“ impliziert (Keygnaert et al. 2012). Schließlich handelt es sich vielfach um Fälle, in denen jungen Flüchtlingen das angegebene Alter nicht (ohne weiteres) geglaubt wird. Zudem findet sich u. U. das Phänomen der ausbleibenden ärztlichen Hilfeleistung - namentlich in Situationen, in denen Röntgenbefunde des Kiefers und der Zähne behandlungsbedürftige Zustände aufweisen, diese aber mit Ausnahme akuter Schmerzen in der Regel (erst einmal) nicht therapiert werden - während andererseits eine zahnärztliche Altersabschätzung als erforderlich angesehen wird (Bozorgmehr und Razum 2015; Ramsthaler et al. 2009; Van Ginneken 2015). Schaden wird schließlich auch durch die Exposition mit ionisierenden Strahlen zugefügt, was dem ärztlichen Grundprinzip der Nonmalefizienz (primum non nocere) immer dann zuwiderläuft, wenn keine gravierenden Gründe für die Durchführung vorliegen (Thevissen et al. 2012). Dieser Aspekt erscheint umso problematischer, als die High Commission for Refugees der UN (UNHCR) explizit die Vermeidung jeglicher Verletzung der körperlichen Integrität von minderjährigen Flüchtlingen fordert (Fang et al. 2015, vgl. dazu Aynsley-Green et al. 2012). Fachlich vorrangig in der Begegnung zwischen Zahnärztin bzw. Zahnarzt und jungem Flüchtling wäre somit eine zahnärztliche Grundversorgung (Den Exter et al. 2001; Jensen et al. 2014), zu der auch Schmerztherapie, Zahnerhalt, suffizienter Zahnersatz und Prophylaxe- 
maßnahmen zählen (Groß 2012). Bisher liegen Studien vor, die nicht dezidiert für den Bereich der Zahnheilkunde, aber für den Gesamtbereich der Medizin belegen, dass eine Maximalversorgung von Flüchtlingen nicht nur aus humanitären Gründen wünschenswert wäre, sondern auch langfristig Kosten für die Gemeinschaft einspart (Bozorgmehr und Razum 2015; Van Ginneken 2015). Aus ethischer und ökonomischer Perspektive ist dies umso beachtlicher, als Studien belegen, dass insbesondere die Zahngesundheit bei $50 \%$ der jungen Flüchtlinge ein gravierendes Problem darstellt und eine entsprechend große Gruppe einer Behandlung bedürfte (Thevissen et al. 2012).

Vor diesem Hintergrund ist es Ziel dieses Beitrages, die bisher wenig diskutierte Rolle der Zahnmedizin in den Verfahren der Altersschätzung kritisch zu beleuchten und eine normative Klärung des Problems zu erarbeiten. Dabei bietet die uneingeschränkte Befürwortung der dentalen Altersbestimmung durch die entsprechende Deutsche Fachgesellschaft den Anlass, die international kritisch und distanziert geführte Debatte zur Altersbestimmung mit dem positiven Votum der Fachgesellschaft zu kombinieren, um die bisherige internationale Diskussion zu konkretisieren und anzuwenden. Darüber hinaus soll ein Beitrag dazu geleistet werden, den innerdeutschen Streit über die Altersbestimmung, der unten angeführt wird, besser einzuordnen. Methodische Grundlagen des Beitrages sind eine umfassende Auswertung der verfügbaren Fachliteratur und eine normative Analyse der maßgeblichen klinisch-ethischen Argumentationslinien. Hierbei wird zunächst das übliche radiologische Verfahren zur Altersbestimmung in der Zahnmedizin vorgestellt. Es folgt eine Analyse des bisher in Deutschland geführten Diskurses über die Eignung, Erforderlichkeit und ethische Angemessenheit röntgenbasierter zahnärztlicher Altersschätzungen. Geltend gemachte Argumente innerhalb dieses Dreischritts sollen dabei kritisch analysiert werden. Am Ende steht eine kritische Diskussion über die Frage der Sinnhaftigkeit einer Altersfestsetzung von Asylsuchenden bzw. eines rigiden Festhaltens an einer festen Altersgrenze und den sich hieraus ergebenden ethischen Problemen.

\section{Der Stellenwert der Panoramaschichtaufnahme in der zahnärztlichen Altersdiagnostik}

Aus forensischer Perspektive gibt es diverse Anlässe für eine Altersbestimmung bei Lebenden und Toten (Corradi et al. 2013). Dabei kommt der Analyse von Knochen (Handwurzelknochen und Schlüsselbein) und Zähnen (forensische Odontologie) seit den 1990er-Jahren eine entscheidende Bedeutung zu (Corradi et al. 2013; Dedouit et al. 2015; Focardi et al. 2014). Die Zahnmedizin leistet einen wesentlichen Beitrag zur Altersbestimmung, da idiosynkratische Zustände der Pathologie und Physiologie des Zahns auch Auskunft über das Alter eines Menschen geben. Insbesondere im Zusammenhang mit Adoption, juveniler Delinquenz und im Umgang mit Flüchtlingen spielt die Altersbestimmung eine wichtige Rolle (Deitos et al. 2015; Thevissen et al. 2012).

Zahnmedizinisch stehen dabei insbesondere die dritten Molaren $\left(3^{\text {rd }} M\right)$ und ihre Mineralisation im Fokus (third molar maturity index) (Cameriere et al. 2008; 
Dedouit et al. 2015; Focardi et al. 2014). Der Fokus liegt auch deshalb auf den Zähnen, weil sie aufgrund ihres späten Durchbruchs in die Mundhöhle weniger durch äußere Faktoren (wie Stress, Fehlernährung etc.) veränderbar sind und somit ein vergleichsweise wenig affizierbares Gewebe des menschlichen Körpers zur Verfügung steht (Deitos et al. 2015). Insbesondere Demirjian et al. (1973) haben dazu anerkannte Grundlagenforschung geleistet, auf der die heutigen zum state of the art gehörenden Arbeiten und Klassifizierungen von Cameriere et al. (2008) aufbauen. Eine exakte Altersdiagnostik bietet die Analyse der Entwicklung der dritten Molaren zur Bestimmung von Volljährigkeit nach Cameriere allerdings nicht, auch wenn sie bei bestimmten anatomischen Umständen vergleichsweise sichere Ergebnisse liefern kann (Cameriere et al. 2008).

Trotzdem kommen die deutschsprachigen Fachgesellschaften zu einer beinahe uneingeschränkten Affirmation der radiologischen Praxis der Altersbestimmung (AGFAD der Deutschen Gesellschaft für Rechtsmedizin 2015; DGZMK 2001). Anders erscheint dies in internationaler Perspektive: Im europäischen Vergleich ist eine deutliche Zurückweisung radiologischer Diagnostik mit Panoramaaufnahmen des Gebisses zur Altersbestimmung notabel (Focardi et al. 2014), Deutschland bildet hier eine Ausnahme. Stellvertretend sei die strikte Zurückweisung von Röntgenaufnahmen allein zur Altersbestimmung durch das Royal College of Radiologists in London genannt, das sowohl 1996 als auch 2007 betonte, dass der Gebrauch von Röntgenstrahlung zu nichtmedizinischen Zwecken inakzeptabel sei. Zudem wird vor einer Verharmlosung der Röntgenstrahlen gewarnt (Focardi et al. 2014; vgl. auch Crawley 2007).

Derzeit gibt es keine sichere und adäquate Methode, um das Alter von (adoleszenten) Menschen exakt zu bestimmen (Michie 2005). Altersbestimmungen erscheinen höchstens näherungsweise möglich zu sein - mit einer unübersehbaren Tendenz $\mathrm{zu}$ willkürlichen und wissenschaftlich nicht legitimierbaren Ergebnissen (Riedelsheimer und Wiesinger 2004). Die in der Einleitung aufgeworfene Frage nach der Geeignetheit, Erforderlichkeit und Angemessenheit einer radiologischen Befundung des Zahnbestandes junger Flüchtlinge wird trotz einiger nicht zu übergehender positiver Einschätzungen grosso modo meist negativ beschieden. Besonders die dentale Altersschätzung erfährt dabei Kritik aufgrund des erhöhten Risikos für eine Manifestation von Schäden durch Röntgenstrahlen bei Kindern und Jugendlichen, da ihr Gewebe noch viel Zeit hat, Tumore zu entwickeln (Ramsthaler et al. 2009).

\section{Die bisher in Deutschland geführte Debatte über zahnmedizinische Altersschätzung}

Die medizinethische Debatte zur Rolle der Zahnärztin und des Zahnarztes im Zusammenhang mit radiologischen Befundungen zur Altersentwicklung junger Flüchtlinge kann derzeit keineswegs als abgeschlossen gelten (Thevissen et al. 2012). Allerdings zeichnet sich innerhalb der organisierten Ärzteschaft eine Tendenz zur Ablehnung der Praxis der Altersbestimmung ab, insbesondere wenn dabei Untersuchungen des Intimbereichs und die Verwendung von Röntgenstrahlen angedacht sind - dies zeigen noch zu erwähnende Beschlüsse im Rahmen der Deutschen Ärztetage 
und eine aktuelle Stellungnahme der Zentralen Ethikkommission der Bundesärztekammer (Bundesärztekammer 2016). Einige deutsche Fachgesellschaften vertreten allerdings den Einsatz röntgenologischer Diagnostik des Alters mit Nachdruck und wenden sich gegen eine Kritik dieser Praxis (Rudolf und Schmidt 2015). Die „Arbeitsgemeinschaft für Forensische Altersdiagnostik“ (AGFAD) der „Deutschen Gesellschaft für Rechtsmedizin“ etwa verwahrt sich gegen die Verbreitung ,wiederholt vorgetragener unzutreffender Behauptungen“"im Zusammenhang mit der Altersdiagnostik, ohne allerdings an entsprechender Stelle auf die vorgebrachten medizinischen und medizinethischen Fragen einzugehen (AGFAD der Deutschen Gesellschaft für Rechtsmedizin 2015). Für den zahnmedizinischen Bereich ist darauf hinzuweisen, dass die „Deutsche Gesellschaft für Zahn-, Mund- und Kieferheilkunde“ (DGZMK) 2001, und dann noch einmal leicht überarbeitet 2008, eine „Empfehlung für die Altersdiagnostik bei Lebenden im Strafverfahren“ verabschiedet hat, in der uneingeschränkt von der Praktikabilität und dem Nutzen von ,in aller Regel Panorama-Schichtaufnahmen“ ausgegangen wird. Dabei wird betont: „Zur Erhöhung der Aussagesicherheit und der Erkennung altersrelevanter Entwicklungsstörungen sollten alle genannten Methoden [körperliche Untersuchung, inkl. Untersuchung der ,sexuellen Reifezeichen“, Röntgen der linken Hand und des Gebisses, Anm. d. Verf.] eingesetzt werden“"(DGZMK 2001). Kritische Hinweise werden nicht aufgenommen, weder hinsichtlich des Patientenschutzes noch mit Blick auf wissenschaftliche Validität. Dies ist umso beachtlicher, als Deutsche Ärztetage 1995, 2007 , 2008, 2009 und 2014 eine Ablehnung der Beteiligung von Ärztinnen und Ärzten an der Feststellung des Alters beschlossen haben (vgl. etwa 113. Deutscher Ärztetag 2010, Ärztetag-Drucksache Nr. V-93) (Gerst 2015; Püschel 2015); was im Übrigen in einem auf der Homepage der AGFAD empfohlenen und zum Download bereit gehaltenen Aufsatz als ideologisch motiviert eingeordnet wird (Schmeling et al. 2008). Dagegen hat der amtierende Präsident der Bundesärztekammer (B ̈̈K), der Radiologe Frank Ulrich Montgomery, Ärztinnen und Ärzten jüngst von einer derartigen Altersbestimmung abgeraten, da u. a. die Validität der Röntgenbefundung zur Altersdiagnostik ungesichert sei (Hillienhof 2015). Auch die bereits genannte Stellungnahme der Zentralen Ethikkommission bei der Bundesärztekammer kommt zu einer ähnlichen Empfehlung (Bundesärztekammer 2016). Ganz anders sah dies in einer Replik gegen Montgomery der Rechtmediziner Klaus Püschel, der offenbar keine Zweifel an der wissenschaftlichen Validität der Methode hegt und betont, es sei eine genuine Aufgabe der Medizin und Zahnmedizin, entsprechende Röntgenuntersuchungen durchzuführen (Püschel 2015). Darauf wird zurückzukommen sein, da offenbar Püschels Position übersieht, dass die wissenschaftliche und medizinethische Basis für Altersschätzungen insgesamt erodiert (Presseerklärung des interdisziplinären DFG-Netzwerks „Kindeswohl und Kinderschutz - Normative Grundlagen des Kindeswohls“ 2016). 


\section{Kritische Analyse der Debatte über die Geeignetheit, Erforderlichkeit und ethische Angemessenheit der zahnmedizinischen Altersschätzung}

Diese Arbeit will einen Beitrag zur weiteren Aufklärung der Debatte über röntgenologische Altersschätzung liefern, insofern sie nach der Geeignetheit, Erforderlichkeit und ethischen Angemessenheit der radiologischen Altersschätzung in der Domäne der Zahnmedizin fragt. Für eine umfassende Bewertung des in Rede stehenden Verfahrens sind alle drei genannten Fragerichtungen konstitutiv: Das Kriterium der Geeignetheit zielt dabei auf die technologisch-diagnostischen Grundlagen der zahnmedizinischen Altersschätzung und diskutiert den zahnmedizinischen state of the art in dieser Sache. Das Kriterium der Erforderlichkeit rückt diagnostische Möglichkeiten aber auch Grenzen in einen weiteren Fokus und thematisiert das Ziel zahnmedizinisch-diagnostischen Könnens. Geht es dem Kriterium der Geeignetheit also um die Frage des Wie in einem engeren Sinne, sondiert das Kriterium der Erforderlichkeit die nicht zu suspendierende Frage nach dem Wie in einem weiteren Sinne. Hier geht es um mögliche Alternativen, insbesondere weniger invasive, die Zahnmedizinerinnen und Zahnmediziner im Dialog mit ihren Patientinnen und Patienten, aber auch mit Blick auf Kostenträger und gesamtgesellschaftliche Erfordernisse sondieren, um ein bestimmtes Ziel zu erreichen. Ob aber die möglichen Ziele auch angemessen sind, verweist zahnmedizinisches Handeln in den Raum ethischer Diskussion, denn allein die diagnostisch-therapeutische Machbarkeit (Kriterium 1) und die nicht willkürliche Fokussierung eines bestimmten Zieles (Kriterium 2), enthält noch keine Aussage über das moralisch ausweisbare Gute oder Schlechte einer entsprechenden Operation (Kriterium 3). Der so plausibilisierte Dreischritt soll im Folgenden für die dentale Radiologie im Zusammenhang der Altersbestimmung wenigstens in seinen zentralen Aspekten durchgeführt werden:

\section{Kriterium 1 - Geeignetheit}

Bewusst ist hier nur von einer „Schätzung“ und nicht von einer Bestimmung des Alters die Rede, weil das biologische Alter allenfalls näherungsweise geklärt werden kann (Aynsley-Green et al. 2012; Bundesärztekammer 2016; Nowotny et al. 2014; Thevissen et al. 2012). Tatsächlich muss bei den erhobenen Befunden von nicht unerheblichen interindividuellen Schwankungen ausgegangen werden, die eine exakte Altersfeststellung a priori unmöglich machen (Michie 2005). Diese Schwankungen bei dentaler Altersdiagnostik ergeben sich, so ein Konsens in der entsprechenden Forschung, aus erheblichen individuellen Variabilitäten (Aynsley-Green et al. 2012; Crawley 2007), insbesondere im Zustand der dritten Molaren, die in der zahnärztlichen Altersschätzung besonders fokussiert werden (Aynsley-Green et al. 2012). $\mathrm{Zu}$ einer hohen individuellen Variabilität kommen ethnologische und sozioökonomische Variabilitäten. Einzelne Studien konnten für bestimmte Ethnien nachweisen, dass jeweils mit erheblichen Abweichungen bei der Altersschätzung zu rechnen ist, insbesondere wenn als Referenzpunkt ein europäischer Durchschnitt angelegt und mit Populationen junger Asylsuchender verglichen wird, die regelmäßig aus nichteuropäischen Kontexten stammen (Aynsley-Green et al. 2012; Crawley 2007). Außerdem herrscht gerade in der international geführten Fachdebatte über die Validität 
der verschiedenen Verfahren der Altersdiagnostik (vom Gespräch mit betreuenden Sozialarbeiterinnen bzw. Sozialarbeitern oder Allgemeinmedizinerinnen bzw. Allgemeinmedizinern zur Feststellung der psychischen Reife (Crawley 2007), Inspektion der körperlichen Reife, mit besonderem Fokus auf die Entwicklung der Geschlechtsmerkmale und -organe bis hin zur Bildgebung der Handwurzelknochen, der Sternoclavikulargelenke und des Kiefers) Konsens darüber, dass auch das Gesamte dieser Methoden eine sichere Altersbestimmung nicht ermöglicht. Die eine Methode kann also nicht die Schwächen der anderen ausgleichen (Crawley 2007), sondern im Gegenteil, die (berufs-)ethischen Probleme würden durch vermehrte Diagnostik noch verschärft (Aynsley-Green et al. 2012). Auch vor diesem Befund scheint es weiter angezeigt, lediglich von einer Altersschätzung auszugehen; auch wenn holistischere Ansätze, die kulturelle und entwicklungspsychologische Aspekte neben anatomischen einbeziehen, als adäquatere Methode diskutiert werden (Aynsley-Green et al. 2012). Der in der gesamten Forschung zur Altersschätzung zu findende negative Bescheid zur Angemessenheit üblicher Verfahren, zu denen auch die zahnärztliche Radiologie gehört, ist umso frappierender, als bei üblichen, auch röntgenologischen Befundungen des Alters von einer Abweichung von ca. zwei bis drei Jahren ausgegangen wird (Nuzzolese und Di Valla 2008). In der Regel kann somit keine sichere Differenzierung zwischen Minder- oder Volljährigkeit erfolgen. Kritisch zu kommentieren ist zudem das Risiko der Fehlbeurteilung des Alters. Es gibt zwar keine exakten Zahlen über nachweisliche Fehleinschätzungen durch Altersbestimmung, doch finden sich anwaltschaftliche Widersprüche gegen allzu evidente Fehldiagnosen, insbesondere wenn 15- oder 16-Jährige für volljährig erklärt werden (Nowotny et al. 2014).

Außerdem stellt der oft problematische psychologische Zustand junger Asylsuchender Anfragen an die Geeignetheit einer radiologischen Altersbestimmung. Gegen medizinisch nicht-indizierte Diagnostik spricht auch der psychische Zustand vieler junger Flüchtlinge, die nach Traumatisierungen unter Angstzuständen, depressiven Symptomen und Verhaltensproblemen leiden. Die Konfrontation mit medizinischer Diagnostik und Apparaturen kann bestehende Traumata verstärken oder - bei überwunden geglaubten Krankheitszuständen - retraumatisierend wirken (Crawley 2007; Jensen et al. 2014).

\section{Kriterium 2 - Erforderlichkeit}

Die High Commission for Refugees der Vereinigten Nationen (UNHCR) empfiehlt im Umgang mit jungen Flüchtlingen, im Zweifelsfall das jüngere Alter anzunehmen, damit den Betroffenen ein umfassender Schutz gewährt wird (Michie 2005; Riedelsheimer und Wiesinger 2004). Dies entspricht in einem weiteren Sinne auch der Logik der UN-Kinderrechtskonvention, die rät, im Zweifel stets für das Wohl des Kindes zu entscheiden (Art. 3) (Riedelsheimer und Wiesinger 2004). Diese Im-Zweifelfür-die-Person-Logik des benefit-of-the-doubt-argument wird umso dringlicher, als die große Anzahl zu begutachtender Personen vielfach einen Entscheidungsdruck hervorrufen, der es Gutachtern u. U. kaum ermöglicht, ein umfassendes Bild von der Reife eines jungen Menschen zu gewinnen (Michie 2005). Schutzdefizite für junge, unbegleitete Flüchtlinge sollten Gutachter überdies animieren, deutlich zu- 
gunsten der besseren Betreuung zu entscheiden (Crawley 2007; Riedelsheimer und Wiesinger 2004).

Vor dem Hintergrund dieser Empfehlungslage gerät das Kriterium der Erforderlichkeit erheblich unter Druck. Verschärfung findet dies, wenn man die Erforderlichkeit einer präzisen Altersschätzung im Kontext von Asylverfahren mit anderen Rechts- und Verwaltungserfordernissen vergleicht, in denen zwar auch das Alter keine unerhebliche Rolle spielt, dabei aber eine klare Priorisierung des nicht-chronologischen Individualalters zu finden ist (Crawley 2007).

So ist in der Rechtspraxis die Unterscheidung zwischen chronologischem und psychischem Alter üblich. Diese Unterscheidung wird in den meisten Fällen zugunsten von jungen Delinquenten zur Anwendung gebracht, die zwar chronologisch volljährig sind, in ihrem Denken und Handeln aber Verhaltensweisen aufweisen, die eher juvenil erscheinen. Strafmaß und andere begleitende Maßnahmen orientieren sich an eben dieser Einordnung. Die gesamte Debatte um die normative Zulässigkeit radiologischer Befundung zur Altersschätzung würde entschärft, wenn, wie im Strafrecht, die einzelne Person im Mittelpunkt stünde und individuelle Reife sowie Bedürftigkeit und nicht allein das biologische Alter ausschlaggebend wären (Nowotny et al. 2014). Demnach wäre es im Hinblick auf die zu gewährenden Schutzrechte unmaßgeblich, ob es sich um eine 17-, 18- oder 21-jährige Person handelt, die ihre Heimat verlassen hat und in diesem Zusammenhang nicht selten traumatisierenden Erlebnissen ausgesetzt war (Stotz et al. 2015): In jedem Fall geht es hierbei um einen jungen Menschen, der zu einer besonders vulnerablen Gruppe gehört und mithin besonderen Schutz verdient, der nicht abrupt mit dem 18. Lebensjahr obsolet wird (Michie 2005). Der gesetzlich geregelte Schutz für die vulnerable Gruppe junger Flüchtlinge ohne Begleitung ist bei Licht besehen nur glaubwürdig, wenn er sich tatsächlich an der konkreten Bedürftigkeit ankommender junger Menschen und nicht an einem Stichtag orientiert. (Zahn)ärztliches Handeln, das sich an Verfahren der Altersschätzung beteiligt, wird dadurch in einem besonderen Maße politisch und bedarf auch vor diesem Hintergrund einer (selbst)kritischen Reflexion (Schmiedebach 2006). $\mathrm{Zu}$ fordern wäre demnach eine Kultur des Augenmaßes - gerade im Umgang mit jungen Menschen, die u. U. traumatisierende Erfahrungen von Krieg, Folter, Flucht, Tod von Angehörigen, Gewalt und/oder Missbrauch durchlebt haben (Michie 2005; Riedelsheimer und Wiesinger 2004; Stotz et al. 2015).

\section{Kriterium 3 - ethische Angemessenheit}

Der im Folgenden dargelegte letzte Punkt der ethischen Argumentation ist weniger daran interessiert, die Debatte um zahnärztliche Altersschätzung durch Röntgen in die üblichen Formate der Medizinethik einzufügen, die entweder einen Four-Principles-Approach favorisieren oder eine Zuordnung zu deontologischen oder konsequentialistischen Ansätzen vorziehen; was im Übrigen auch bereits geschehen ist (vgl. beispielsweise Aynsley-Green et al. 2012). Gerade aufgrund des besonderen Praxisbezugs der hier zugrunde liegenden Problematik scheint es sinnvoller, auf solche Aspekte aufmerksam zu machen, die unabhängig von bestimmten frameworks als Indikatoren für ethische Probleme fungieren. Dass dabei implizit auf ethische Theorien zurückgegriffen wird, wird an entsprechenden Stellen notiert. 
Mögliche Güterabwägungen, die den Schutz der Person vor bloßstellenden Untersuchungen und gesundheitsschädlicher Strahlung niedriger bewerten als Belange der geforderten Altersbestimmung, lassen sich unter Umständen als eine Form von Fetischismus begreifen, so wie ihn Simon Keller (2009, S. 90) für ethische Argumentationen definiert hat: "A moral view is fetishist when it takes something that has merely derived moral importance and treats it as though it has fundamental moral importance.” Das Fetischismus-Argument gilt dann für die radiologische Altersbestimmung, wenn man der Überzeugung ist, dass die Altersbestimmung nicht im Dienste eines sonderlich schützenswerten Gutes steht und die Applikation ionisierender Strahlen als unverhältnismäßig erscheinen muss. In dieser Optik bestünde das Moment des Fetischistischen darin, etwas mit lediglich aspektiver Bedeutung, hier die (radiologische) Altersschätzung, in das Zentrum der Wahrnehmung, hier des jungen Asylsuchenden, zu rücken.

Neben diesem Beispiel für eine mögliche Fundamentalkritik der Altersfestsetzung finden sich kritische Einzelaspekte, die in einer ethischen Sondierung besonderes Gewicht haben. So erscheint etwa der Hinweis, der auf die freiwillige Zustimmung von jungen Flüchtlingen zur Röntgendiagnostik zur Altersschätzung abhebt (Dedouit et al. 2015), als mitunter zynisch. Zum einen geschieht dieses decision making in einem Kontext, in dem Betroffene bedürftig, abhängig und insofern vulnerabel sind. So gibt es Hinweise darauf, dass eine Nicht-Einwilligung in eine Röntgenbefundung, außer im Falle einer Schwangerschaft, negative Konsequenzen hat und als fehlender Kooperationswille ausgelegt werden kann (Thevissen et al. 2012). Zum anderen zeigen psychologische Untersuchungen an jungen Flüchtlingen eine Trauma-induzierte Schamhaftigkeit, die zugleich mit Schuldgefühlen assoziiert ist. Auch Angst vor Isolation und gesellschaftlicher Ausgrenzung ist in der Gruppe junger Flüchtlinge ohne Begleitung in signifikantem Umfang nachzuweisen. In dieser schwierigen Situation kann jungen Flüchtlingen kaum Opposition gegen diejenigen zugemutet werden, bei denen sie Zuflucht suchen.

Auch der Hinweis auf die relativ geringe Strahlenbelastung durch Röntgen von Hand und Kiefer sowie durch ein CT des Schlüsselbeins, kann nicht als ethische Legitimationsfigur für die Schätzung des Alters durch ionisierende Bildgebung herhalten. Als Kritikpunkt gegenüber den radiologischen Verfahren zur Altersschätzung wird besonders, wie bereits genannt, die Strahlenexposition angeführt. Zwar liege diese bei vollumfänglicher Altersdiagnostik mit ca. 800 Mikrosievert nicht sehr hoch, kommt aber dennoch bereits nahe an den Grenzwert für künstliche Strahlenexposition heran, der für ein gesamtes Jahr bei 1000 Mikrosievert liegt (Nowotny et al. 2014; Strong et al. 2015). Immerhin handelt es sich also um die Inkaufnahme potentieller Schädigungen für eine fragwürdige, weil nicht medizinisch indizierte Befundung. So fällt ein temporäres Desinteresse an der körperlichen Integrität auf. Einen jungen Flüchtling ohne medizinische Indikation ionisierenden Strahlen auszusetzen, impliziert mithin nicht nur ein „Ich glaube Dir nicht“" (Crawley 2007), sondern auch ein - für das Berufsethos noch gravierender - „Ich helfe Dir nicht“" (wenn die Begegnung allein aus gutachterlichen Gründen erfolgt), sowie ein dem

\footnotetext{
${ }^{1}$ Denn sonst würde man die Altersangabe der betroffenen Person übernehmen, und als Ausnahmen verblieben nur solche Migrantinnen und Migranten, die selber keine Auskunft geben können.
} 
Berufsethos gänzlich zuwiderlaufendes „Ich füge Dir Schaden zu“. Auch im Fall der Bildgebung von Kiefer, Handgelenk und Schlüsselbein, bleibt es nicht allein bei einer symbolisch vermittelten Erniedrigung, vielmehr steigt mit jeder unnötigen Strahlenexposition das Krebsrisiko (Nowotny et al. 2014). Hinzu kommt die ohnehin schwierige medizinische Versorgung von Flüchtlingen (Fang et al. 2015; Pottie et al. 2015), so dass der Kontakt mit Zahn- und Humanmedizinerinnen bzw. -medizinern und einer High-Tec-Gerätemedizin umso kritischer gesehen werden muss, wenn sie dem Einzelnen keinen medizinischen Nutzen bringt (Dedouit et al. 2015).

Fraglich ist, ob Debatten um potentielle Schädigungen durch Röntgenstrahlung zur Altersschätzung, Diskussionen um eine Altersabweichung von einigen Jahren und Rechte für die betroffenen jungen Menschen, die Gewalt, Krieg und Katastrophen erlebt und überlebt haben, nicht ohnehin belanglos erscheinen werden. Das mag zwar individuell tatsächlich so erlebt werden, insbesondere bei einem nicht selten zu erwartenden hohen Grad der Traumatisierung. Die bereits angeführte stets auch symbolische Dimension der Begegnung zwischen (zahn)medizinischem Personal und jungen Asylsuchenden macht es möglicherweise aber gerade erforderlich, den Ort des erhofften Schutzes durch detaillierte Sorgsamkeit gegenüber jungen Asylsuchenden und ihrer körperlichen Integrität zu bewähren.

Außerdem stellt sich die bereits intonierte Frage nach dem Berufsethos von Zahnärztinnen und Zahnärzten, denn zur Altersdiagnostik werden zahnmedizinische Kenntnisse und Fertigkeiten nicht auf gesundheitsrelevante Befunde und das Wohlergehen von Patientinnen und Patienten gerichtet. Stattdessen wird von Zahnmedizinerinnen und -medizinern verlangt, ihre Kompetenz für fachfremde Ziele anzuwenden, um dabei mindestens die beiden genannten Kernbereiche ihres Handelns (zahngesundheitsrelevante Befundung und das Wohlergehen von Patientinnen und Patienten) wenigstens passager zu übergehen (Crawley 2007).

Auch die Rolle der Rechtsmedizin und die forensische Aufgabe der Begutachtung erscheinen in der Diskussion um die Schätzung von Minderjährigkeit oder Volljährigkeit in ethischer Hinsicht klärungsbedürftig. Zwar gehört die Begutachtung aus amtlichen Gründen zu ihren Aufgaben, dies entbindet sie aber nicht von der ärztlichen Verpflichtung auf das Nichtschadensgebot. Anders als bei einer anamnestischen oder rein körperlichen Untersuchung und Begutachtung wird im Fall der röntgenologischen Diagnostik ein Gesundheitsrisiko in Kauf genommen. Das in der aktuellen Debatte deutlich gewordene Insistieren einiger deutscher Rechtsmedizinerinnen und Rechtsmediziner auf die radiologische Altersbestimmung ist - wie oben gezeigt nicht auf der Höhe der internationalen Forschung in diesem Bereich. Besagte Position verkennt zudem, dass die Rechtsmedizin, ähnlich wie die Psychiatrie, eng an gesellschaftliche Entwicklungen gekoppelt ist und insofern einer fortgesetzten gesellschaftlichen - und normativen - Legitimierung bedarf (Pollak 2004; Schmiedebach 2005). Ein Legitimationsformat der Altersbestimmung, das nicht nach der Moralität, sondern allein nach der momentanen Legalität fragt, offenbart eine Praxis, die sich mehr an Gewohnheiten und weniger an begründungsfähigen ethischen Argumenten orientiert (Püschel 2015). 


\section{Ausblick und Praxis}

Der Einsatz von Röntgendiagnostik zur Altersbestimmung von minderjährigen, unbegleiteten Flüchtlingen führt in der Praxis der begutachtenden Zahnärztin bzw. des Zahnarztes, der Kieferorthopädin bzw. des Kieferorthopäden oder der Gerichtsmedizinerin bzw. des Gerichtsmediziners regelmäßig, wenn auch eher unbewusst bzw. unreflektiert, zum Mechanismus des othering des ohnehin Fremden. Das mag in vielen Gutachtersituationen in der Natur der Sache zu liegen, doch im gutachterlichen Umgang mit jungen, minderjährigen Flüchtlingen bedeutet othering zugleich eine Art déformation professionnelle, da junge, unbegleitete Flüchtlinge von Ärztinnen und Ärzten gemäß deren Heilauftrag primär Schutz und Hilfe erwarten dürfen - und eben nicht eine gutachterlich-bürokratische Überprüfung ihrer Altersangaben. Ordnungspolitische Maßnahmen dürfen nicht wichtiger werden als die Beantwortung individueller Bedürfnisse (Pottie et al. 2015).

Dem entspricht eine am Wohlergehen der Person orientierte Ethik (z. B. der welfarism), die eine Handlung insofern gut nennt, als sie das Wohl des Individuums fördert. Moralische Subjekte in diesem Sinn sind alle, denen es besser oder schlechter gehen kann, und Moral ist ,,[...] centrally concerned with the best interest of individuals“ (Keller 2009). Besonders überzeugend an einer solchen Konzeption ist, dass es in der Tat keine Moraltheorie und keine Bereichsethik, wie etwa die Ethik der Zahnheilkunde, geben kann, in der nicht an zentraler Stelle das individuelle Wohlergehen steht (Giuseppa 2009; Schmiedebach 2006, 1999). Somit wird deutlich, welche Position eine am Wohlergehen der Einzelperson orientierte Ethik gegenüber dem Problem der Altersdiagnostik einnehmen würde (Keller 2009): eine klar ablehnende, weil gerade nicht das Wohl des Einzelnen fokussiert wird. Ein solches Plädoyer im Zusammenhang mit zahnmedizinischer Altersschätzung könnte auch einen Beitrag zu einer Operationalisierung zahnärztlichen Handelns leisten. Scheint die bisherige Praxis vornehmlich gerechtigkeitstheoretisch grundiert und ihre Legitimation aus der Betrachtung des größeren, gesellschaftlichen Kontextes zu gewinnen, so kann die individuelle zahnärztliche Praxis diese Dimension distributiver Gerechtigkeit zwar nicht völlig unbeachtet lassen, aber doch anders gewichten. Gerade die berufsethisch leicht ableitbare Priorisierung des Individualwohls vor dem Gemeinwohl, die keineswegs beinhaltet, dass Probleme der Verteilung und der Gerechtigkeit gehaltlos wären, impliziert eine Option, wie Zahnmedizinerinnen und Zahnmediziner sich verhalten können, wenn der Staat Altersbestimmungen verlangt. Konkret könnte man stets zur Geltung bringen, eine Befundung nur dann vorzunehmen, wenn sie sich nicht exklusiv auf die Altersschätzung bezieht, da es einem begründeten zahnmedizinischen Ethos widerspräche, relevante Zustände der Zahn- und Kiefergesundheit bewusst zu ignorieren. Dies setzt allerdings voraus, dass man die Altersbestimmung nicht aus oben angeführten Gründen grundsätzlich ablehnt - was aufgrund der Ungeeignetheit der Maßnahme eine vertretbare Position darstellt - da in der Tat Fälle denkbar sind, wo gerade durch eine medizinisch-orientierte Festsetzung des Alters jungen Asylsuchenden zu Rechten verholfen werden kann (Crawley 2007). Bei einem solchen Vorgehen würde man jedenfalls beiden in Rede stehenden Problembezirken gerecht: dem individualethischen Diskurs zahnärztlicher Praxis dadurch, dass man das individuelle Wohl von Patientinnen und Pa- 
tienten priorisiert, dem sozialethischen Diskurs zahnärztlicher Praxis dadurch, dass man in einem untergeordneten Sinne bereit ist, wenn ohnehin eine röntgenologische Befundung indiziert ist, dies auch zum Anlass einer Altersschätzung zu nehmen, insofern der junge Asylsuchende seinem Behandler oder seiner Behandlerin dazu sein Placet erteilt (Crawley 2007).

Das berühmte Diktum von Hans Jonas „Sieh hin und du weißt“ sollte auch für den zahnärztlichen Umgang mit jungen, unbegleiteten Flüchtlingen gelten. Junge, potentiell traumatisierte Flüchtlinge verdienen zumindest dieselbe Achtsamkeit wie jeder andere gedachte Jugendliche - für jeden Einzelnen sollte im Sinne des Verallgemeinerungsgrundsatzes als moralische Richtschnur gelten: "What, if this were my child?" (Michie 2005).

\section{Einhaltung ethischer Richtlinien}

Interessenkonflikt M. Wirth, C.L. Menzel, D. Chan Mi Lee und H.-P. Schmiedebach geben an, dass kein Interessenkonflikt besteht.

Ethische Standards Dieser Beitrag beinhaltet keine von den Autoren durchgeführten Studien an Menschen oder Tieren.

\section{Literatur}

Arbeitsgemeinschaft für Forensische Altersdiagnostik (AGFAD) der Deutschen Gesellschaft für Rechtsmedizin (2015) Aktuelle Diskussion zur Altersdiagnostik bei unbegleiteten fraglichen minderjährigen Flüchtlingen. http://campus.uni-muenster.de/agfad_start.html. Zugegriffen: 23. März 2015

Aynsley-Green A, Cole TJ, Crawley H, Lessof N, Boag LR, Wallace RMM (2012) Medical, statistical, ethical and human rights considerations in the assessment of age in children and young people subject to immigration control. Br Med Bull 102:17-42

Bozorgmehr K, Razum O (2015) Effect of restricting access to health care on health expenditures among asylum-seekers and refugees: a quasi-experimental study in Germany, 1994-2013. PLOS ONE 10(7):e0131483. doi:10.1371/journal.pone.0131483

Bundesärztekammer (2016) Stellungnahme der Zentralen Kommission zur Wahrung ethischer Grundsätze in der Medizin und ihren Grenzgebieten (Zentrale Ethikkommission) bei der Bundesärztekammer: „Medizinische Altersschätzung bei unbegleiteten jungen Flüchtlingen“. Dtsch Ärztebl 113:A1-A6

Cameriere R, Ferrante L, De Angelis D, Scarpino F, Galli F (2008) The comparison between measurement of open apices of third molars and Demirjian stages to test chronological age over 18 years olds in living subjects. Int J Legal Med 122:493-497

Corradi F, Pinchi V, Barsanti I, Manca R, Garatti S (2013) Optimal age classification of young individuals based on dental evidence in civil and criminal proceedings. Int J Legal Med 127:1157-1164

Crawley H (2007) When is a child not a child? Asylum, age disputes and the process of age assessment (= ILPA research report). http://www.ilpa.org.uk/data/resources/13266/ILPA-Age-Dispute-Report. pdf. Zugegriffen: 9. Aug 2016

Dedouit F, Saint-Martin P, Mokrane FZ, Savall F, Rousseau H, Crubézy E, Rougé D, Telmon N (2015) Virtual anthropology: useful radiological tools for age assessment in clinical forensic medicine and thanatology. Radiol Med. doi:10.1007/s11547-015-0525.1

Deitos AR, Costa C, Michel-Crosato E, Galić I, Cameriere R, Biazevic MG (2015) Age estimation among Brazilians: younger or older than 18? J Forensic Leg Med 33:111-115

Demirjian A, Goldstein H, Tanner JM (1973) A new system of dental age assessment. Hum Biol 45:211-227

Deutsche Gesellschaft für Zahn-, Mund- und Kieferheilkunde (DGZMK) (2001) Empfehlung für die Altersdiagnostik bei Lebenden im Strafverfahren. http://www.dgzmk.de/uploads/tx_szdgzmkdocuments/ Empfehlungen_fuer_die_Altersdiagnostik_bei_Lebenden_im_Strafverfahren.pdf. Zugegriffen: 23. März 2015 
DFG-Netzwerk (2016) Presseerklärung des interdisziplinären DFG-Netzwerks „Kindeswohl und Kinderschutz - Normative Grundlagen des Kindeswohls“. Zur Tagung in Göttingen im Oktober 2016 im Lichtenberg-Kolleg in der Alten Sternwarte. http://www.egmed.uni-goettingen.de/fileadmin/PDF/ Wiesemann/PI-DFG-Netzwerk_Kindeswohl_und_Kinderschutz_Ergebnis_Workshop_Goettingen_ 2016.pdf. Zugegriffen: 23. Okt 2016

Den Exter A, Hermans H, van Krieken P, Toebes P (2001) Right to health. In: Van Krieken P (Hrsg) Health, migration and return. A handbook for multidisciplinary approach. T.M.C. Asser Press, Hague, S 9-17

Fang ML, Sixsmith J, Lawthom R, Mountian I, Shahrin A (2015) Experiencing 'pathologized presence and normalized absence'; understanding health related experiences and access to health care among Iraqi and Somali asylum seekers, refugees and persons without legal status. BMC Public Health 15:923-935

Focardi M, Pinchi V, De Luca F, Norelli GA (2014) Age estimation for forensic purposes in Italy: ethical issues. Int J Legal Med 128:515-522

Gerst T (2015) Randnotiz: Keine ärztliche Aufgabe. Dtsch Ärztebl 112:A-1261

Van Ginneken E (2015) Restricting access to the NHS for undocumented migrants is bad policy at high cost. BMJ. doi:10.1136/bmj.h3056

Giuseppa E (2009) Ethical issues for radiographers: general observations and a pilot qualitative study. Radiat Prot Dosimetry 134:88-89

Groß D (2012) Ethik in der Zahnmedizin. Ein praxisorientiertes Lehrbuch mit 20 kommentierten klinischen Fällen. Quintessenz, Berlin

Hillienhof A (2015) Montgomery mahnt zu menschlichem Umgang mit Flüchtlingen. http://www. aerzteblatt.de/nachrichten/63348/Montgomery-mahnt-zu-menshclichem-Umgang-mit-Fluechtlingen. Zugegriffen: 30. Nov. 2015

Jensen TK, Skardalsmo E, Fjermestad KW (2014) Development of mental health problems - a follow-up study of unaccompanied refugee minors. Child Adolesc Psychiatry Ment Health 8:29-39

Keller S (2009) Welfarism. Philos Compass 4:82-95

Keygnaert I, Vettenburg N, Temmerman M (2012) Hidden violence is silent rape: sexual and gender-based violence in refugees, asylum seekers and undocumented migrants in Belgium and the Netherlands. Cult Health Sex 14:505-520

Michie CA (2005) Age assessment: time for progress? Arch Dis Child 90:612-613

Nowotny T, Eisenberg W, Mohnike K (2014) Unbegleitete minderjährige Flüchtlinge: Strittiges Alter strittige Altersdiagnostik. Dtsch Ärztebl 111:A-786

Nuzzolese E, Di Valla G (2008) Forensic dental investigations and age assessment of asylum seekers. Int Dent J 58:122-126

Pollak S (2004) Clinical forensic medicine and its main fields of activity from the foundation of the German Society of Legal Medicine until today. Forensic Sci Int 144:269-283

Pottie K, Martin JP, Cornish S, Biorklund LM, Gayton I, Doerner F, Schneider F (2015) Access to healthcare for the most vulnerable migrants: a humanitarian crisis. Confl Health 9:16-19

Püschel K (2015) Randnotiz: Selbstverständlich eine ärztliche Aufgabe. Dtsch Arztebl 112:A-1274

Ramsthaler F, Proschek P, Betz W, Verhoff MA (2009) How reliable are the risk estimates for x-ray examinations in forensic age estimations? A safety update. Int J Legal Med 123:199-204

Riedelsheimer A, Wiesinger I (2004) Der erste Augenblick entscheidet. Clearingverfahren für unbegleitete minderjährige Flüchtlinge in Deutschland. Standards und Leitlinien für die Praxis. Von Loeper Literaturverlag, Karlsruhe

Rudolf E, Schmidt S (2015) Wo kommen eigentlich all die Irrtümer her? Unzutreffende Behauptungen über altersbezogene Sachverhaltserhebung im ausländerrechtlichen Kontext. http://campus.uni-muenster. de/fileadmin/einrichtung/agfad/002.pdf. Zugegriffen: 10. Dez. 2015

Schmeling A, Grundmann C, Fuhrmann A, Kaatsch HJ, Knell B, Ramsthaler F, Reisinger W, Riepert T, Ritz-Timme S, Rösing FW, Rötzscher K, Geserick G (2008) Aktualisierte Empfehlung für Altersschätzung bei Lebenden im Strafverfahren. http://agfad.uni-muenster.de/german/empfehlungen/ empfehlunge_strafverfahren.pdf. Zugegriffen: 16. Okt. 2015

Schmiedebach H-P (1999) Patientenrecht, ärztliche Dominanz und die „Bedeutungslosigkeit des Einzelwesens“ - Das Individuum und die Medizin um 1900. In: Joerden JC (Hrsg) Der Mensch und seine Behandlung in der Medizin. Bloß ein Mittel zum Zweck?. Springer, Berlin, S 51-66

Schmiedebach H-P (2005) Der mühsame Weg zur akademischen Disziplin. Anmerkungen zur Geschichte der Rechtsmedizin in Hamburg. In: Freundes- und Förderkreis des Universitätsklinikums HamburgEppendorf e. V. Hamburg (Hrsg) Jahrbuch 2005. Drucksache des Freundes- und Förderkreises des Universitätsklinikums Hamburg-Eppendorf, Hamburg, S 34-42 
Schmiedebach H-P (2006) Politische Positionen und ethisches Engagement: Julius Moses und die Medizin im 20. Jahrhundert. In: Schneider M (Hrsg) Julius Moses. Schrittmacher der sozialdemokratischen Gesundheitspolitik in der Weimarer Republik. Friedrich-Ebert-Stiftung, Bonn, S 9-37

Stotz SJ, Elbert T, Müller V, Schauer M (2015) The relationship between trauma, shame, and guilt: findings from community-based study of refugee minors in Germany. Eur J Psychotraumatol 6:25863

Strong J, Varady C, Chahda N, Doocy S, Burnham G (2015) Health status and health needs of older refugees from Syrian in Lebanon. Confl Health 9:12-22

Thevissen PW, Kvaal SI, Willems G (2012) Ethics in age estimation of unaccompanied minors. J Forensic Odontostomatol 30:85-102 